\title{
Serum Fibroblast Growth Factor 21 Levels Are Inversely Associated with Growth Rates in Infancy
}

\author{
Verónica Mericq ${ }^{a}$ Francesco De Lucad $^{d}$ Maria I. Hernandez ${ }^{d}$ Veronica Peña ${ }^{b}$ \\ Katherine Rossel $^{\mathrm{b}}$ Mirna Garcia ${ }^{\mathrm{b}} \quad$ Alejandra Avila ${ }^{\mathrm{a}}$ Gabriel Cavadac \\ German Iñiguez ${ }^{a}$ \\ ${ }^{a}$ Institute of Maternal and Child Research, Faculty of Medicine, University of Chile, ${ }^{b}$ Neonatology Unit, San Borja \\ Arriaran Hospital, 'Public Health Department, University of Chile and University of Los Andes, Santiago, Chile; \\ dSection of Endocrinology and Diabetes, St. Christopher's Hospital for Children, Drexel University College of \\ Medicine, Philadelphia, Pa., USA
}

\section{Key Words}

FGF21 · Growth factors · Preterms

\begin{abstract}
Background: Fibroblast growth factor 21 (FGF21) is a metabolic and growth regulator. Aim: To investigate the role of FGF21 during growth in infancy. Methods: Blood samples for FGF21, leptin, insulin and glucose were collected from cord blood obtained from 95 preterm and term newborns (crosssectional group), and at 6 and 12 months of life in 80 preterm and term infants (longitudinal group). Length and weight were measured at birth, 6 months, and 12 months. Results: From birth through 12 months of age, preterm infants' linear growth and weight gain were larger than those of term infants, irrespective of birth weight SDS. At birth and at 12 months, there was no difference in FGF21 levels between preterm and term infants; in contrast, at 6 months, serum FGF21 in term infants was significantly higher than that of preterm ones. In the 0-6-month period, in the whole longitudinal group, serum FGF21 was inversely correlated to the length change SDS, and such a significant inverse correlation persisted in the preterm-AGA group in the 6-12-month pe-
\end{abstract}

\section{KARGER}

E-Mail karger@karger.com

www.karger.com/hrp riod. In addition, term infants who experienced length catchup in the first 6 months of life exhibited lower serum FGF21 levels at 6 months, and those with length catch-up growth between 6 and 12 months had a greater decrease of serum FGF21 level in the same time period. Conclusions: Our results indicate that FGF21 in infancy is inversely correlated with linear growth rate, thus suggesting that FGF21 is a negative regulator of human growth.

(c) 2014 S. Karger AG, Basel

\section{Introduction}

Nutrition is one of the most important determinants of statural growth. Indeed, the most common cause of growth failure worldwide is malnutrition. While human and experimental evidence indicate that growth hormone (GH) insensitivity is a major hormonal mechanism underlying malnutrition-related poor growth, yet the molecular signals leading to GH insensitivity are illdefined.

Fibroblast growth factor 21 (FGF21) is a recently discovered member of the FGF family, primarily known to 
regulate multiple metabolic pathways. FGF21 is induced in the liver by fasting and its increased expression/activity leads to increased fatty acid oxidation and ketogenesis [1]. In obese or diabetic animals, FGF21 preserves $\beta$-cell function and mass, ameliorates steatosis, and improves leptin resistance [2]. Recent evidence also indicates that FGF21 may be an important regulator of growth. Transgenic mice overexpressing FGF21 exhibit reduced longitudinal bone growth and GH insensitivity [3]. Furthermore, it has been shown that sustained caloric reduction in mice leads to increased expression of FGF21 both in the liver and in the growth plate, which in turn results in reduced $\mathrm{GH}$ receptor binding and action, possibly through endocrine and paracrine effects of FGF21 [3, 4].

Relatively little is known about the regulation of FGF21 in humans. Several recent reports showed that circulating FGF21 concentrations are increased in adults or adolescents who are overweight, have type 2 diabetes or have impaired glucose tolerance [4]. Interestingly, serum FGF21 levels are also elevated in individuals on a very low calorie diet [4].

In addition, a recent study in girls with anorexia nervosa has shown increased serum FGF21 levels when adjusted for insulin sensitivity and body fat. In this study, FGF21 was positively associated with integrated concentrations of GH, thus suggesting that FGF21 may mediate a state of GH resistance in anorexia nervosa [5]. An earlier study reported lower FGF21 levels in women with anorexia nervosa compared to normal-weight women. However, FGF21 levels were not controlled for insulin resistance and body fat content, variables that are known to impact FGF21 levels [6].

Based on this evidence, we hypothesized that FGF21 concentrations would be inversely related to growth rate in infancy. Specifically, we hypothesized that accelerated linear growth and weight gain, typically experienced by preterm infants during the first 12 months of life, would be associated with reduced serum FGF21 levels.

\section{Subjects and Methods}

All infants were recruited consecutively at birth from the neonatal unit at Hospital San Borja Arriaran. Two groups of infants were included. The first group (cross-sectional group) included 95 newborns whose cord blood was obtained for determinations of FGF21, glucose, insulin, and leptin. 55 of these newborns were the products of term pregnancies and 40 were born preterm (26-35 weeks of gestation).

The second group (longitudinal group) was composed of 80 newborns: 40 of them were products of term pregnancies (37-
40 weeks of gestation) and 40 were born either preterm $(<32$ weeks of gestation) or with very low birth weight (VLBW $<1,500$ g). After enrollment at birth, these 80 infants were evaluated at 6 and 12 months of age by a pediatric endocrinologist at the Institute of Maternal and Child Research, Faculty of Medicine, University of Chile. Length and weight were measured by one nurse (A.A.).

An infant stadiometer was used to measure supine length. Weight was measured using a manual scale with a 10-gram gradation (Seca; QuickMedical, Snoqualme, Wash., USA). At 6 and 12 months, after an overnight fast of $\geq 6 \mathrm{~h}$, a blood sample was obtained for the measurement of FGF21, glucose, insulin, and leptin concentrations. All samples were centrifuged immediately and stored at $-70^{\circ} \mathrm{C}$.

Newborns with a birth weight between the 10th and the 90th percentiles for gestational age were considered as AGA and below the 10th percentile as SGA. Exclusion criteria at birth and/or during the first year of life included medical, neurological, or genetic disorders or treatment with medications known to impair linear/ statural growth. Feeding practices for all the VLBW infants during the first weeks of life and until 1 year of CA have been described elsewhere [7]. One third of the term and preterm newborns were delivered by cesarean section and Apgar score at 5 min of all them was 9 or more.

This study was approved by the institutional review boards of the participating institutions. A parent or guardian of each study infant provided written informed consent.

Assays

Serum FGF21 was measured by ELISA (Millipore Corp., Billerica, Mass., USA). Serum insulin was measured using a commercial immunoradiometric (IRMA) assay and serum leptin by RIA assay. IRMA and RIA kits were purchased from DiaSource Immunoassays SA (Nivelles, Belgium). Intra-assay coefficients of variation (CVs) were $5.6 \%$ for FGF21, 3.8\% for insulin and $4.6 \%$ for leptin. Inter-assay CVs were 7-9\% for FGF21, 4.7\% for insulin and $6.2 \%$ for leptin. In the RIA insulin assay, no cross-reactivity with proinsulin was declared by the manufacturer.

\section{Calculations and Statistical Analysis}

Weight and length at birth were converted into standard deviation scores (SDS) by using local growth curves based on national validated references for term [8] and for preterm growth [9]. Thereafter, anthropometric measurements at 6 and 12 months of age were expressed as SDS according to the WHO growth standards [10]. Weight gain and linear growth were calculated for two time periods: $0-6$ and 6-12 months.

Characteristics and anthropometric data are expressed as median with interquartile range (IQR), percentages, and the effect of infancy growth on later outcomes as estimated regression coefficients $(\beta s)$ with $95 \%$ confidence interval (CI). Significant weight catch-up was defined as a change in weight, between zero and 12 months, $>0.67$ SDS, which represents the width of each percentile band in standard growth charts. Length catch-up was defined as a change in length $>0.67$ SDS during the same period [11]. Growth and metabolic parameters are expressed as median with IQR. Normal data distribution was tested using the Shapiro-Wilk test. Differences between groups were assessed by Student's t test or nonparametric test (Mann-Whitney U test) depending on the normality of the data and exact Fisher's test was 

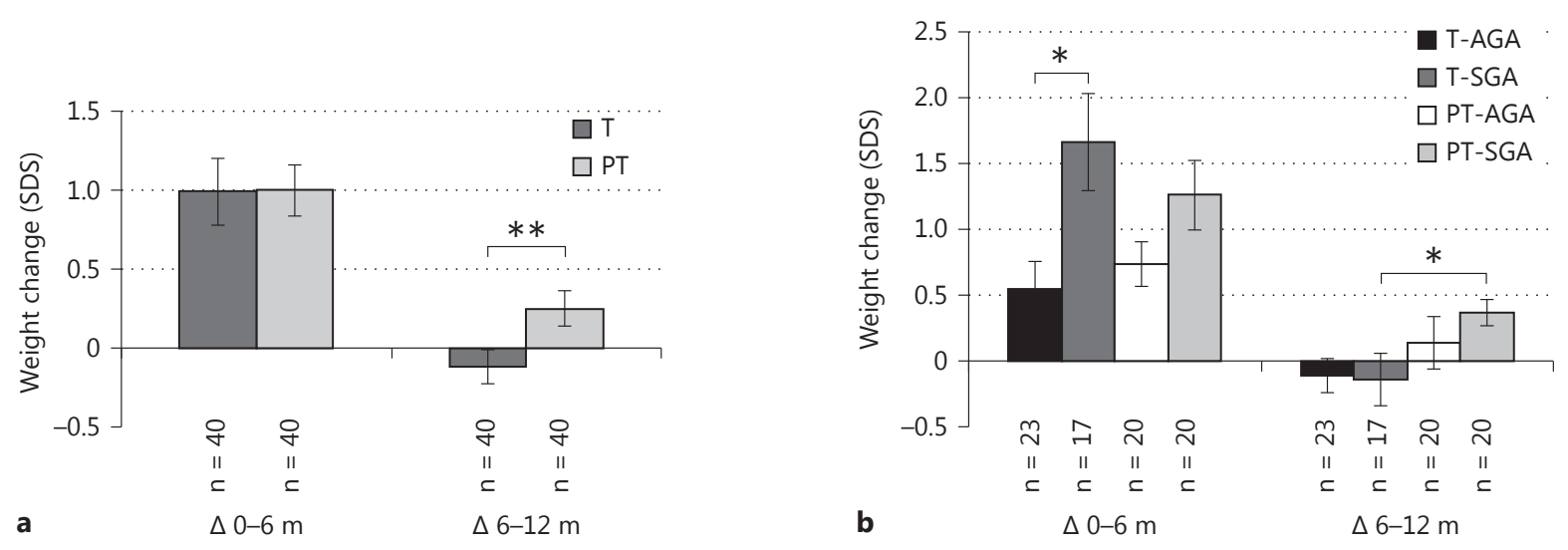

Fig. 1. Postnatal weight SDS changes in the first semester $(\Delta 0-6 \mathrm{~m})$ and in the second semester $(\Delta 0-12 \mathrm{~m})$ of life, comparing the term (T) and preterm (PT) infants (a) and T and PT infants grouped according to by BW SDS (AGA or SGA) (b). Mean \pm SEM. ${ }^{*} \mathrm{p}<0.05$, ** $\mathrm{p}<0.01$.

Table 1. Anthropometric characteristics at birth and gestational age of infants enrolled in the cross-sectional and longitudinal groups

\begin{tabular}{lllll}
\hline & Term-AGA & Term-SGA & Preterm-AGA & Preterm-SGA \\
\hline Cross-sectional group & & & & \\
$\quad$ Number & 28 & 27 & 21 & 19 \\
GA, weeks & $39.3 \pm 1.2$ & $38.3 \pm 1.2$ & $34.4 \pm 3.1$ & $33.2 \pm 3.1$ \\
BW (SDS) & $0.02 \pm 0.83$ & $-1.61 \pm 0.26^{* *}$ & $-0.53 \pm 0.58$ & $-2.03 \pm 0.52^{* *}$ \\
BL (SDS) & $-0.25 \pm 0.90$ & $-1.77 \pm 0.83^{* *}$ & $-0.69 \pm 1.05$ & $-1.76 \pm 0.81^{* *}$ \\
Longitudinal group & & & & \\
Number & 23 & 17 & 20 & 20 \\
GA, weeks & $39.2 \pm 0.2$ & $38.6 \pm 0.3$ & $29.0 \pm 0.4$ & $31.3 \pm 0.5$ \\
BW (SDS) & $0.11 \pm 0.13$ & $-1.98 \pm 0.15^{* *}$ & $-0.91 \pm 0.11$ & $-2.66 \pm 0.15^{* *}$ \\
BL (SDS) & $0.59 \pm 0.19$ & $-1.34 \pm 0.31^{* *}$ & $-0.49 \pm 0.15$ & $-1.95 \pm 0.22^{* *}$ \\
\hline
\end{tabular}

** $\mathrm{p}<0.01$ for comparisons within the same group and same gestational age between those born appropriate (AGA) or small for gestational age (SGA).

used for categorical variables. The correlation between length SDS or weight SDS changes (0-12, 0-6, and 6-12 months), variables and serum concentrations of hormones was evaluated using Spearman's correlation. The temporal evolution of height SDS was associated with the temporal evolution of growth and metabolic parameters by regression analysis for repeated measures estimated through mixed models. Changes in hormonal concentrations between 6 and 12 months were calculated using Kruskal-Wallis test with Bonferroni principle for multiple comparisons. Confidence intervals are level 95 and 5\% significance. Data were analyzed with the statistical package STATA version 12.0 .

\section{Results}

\section{Growth Parameters}

Changes in Weight over Time

Anthropometric characteristics and gestational age of enrolled infants are presented in table 1. Postnatal weight gain and linear growth in the first 12 months of life are presented in figures 1 and 2, respectively. Between 0 and 6 months, we found no difference in weight gain SDS between term and preterm infants (fig. 1a); however, the 

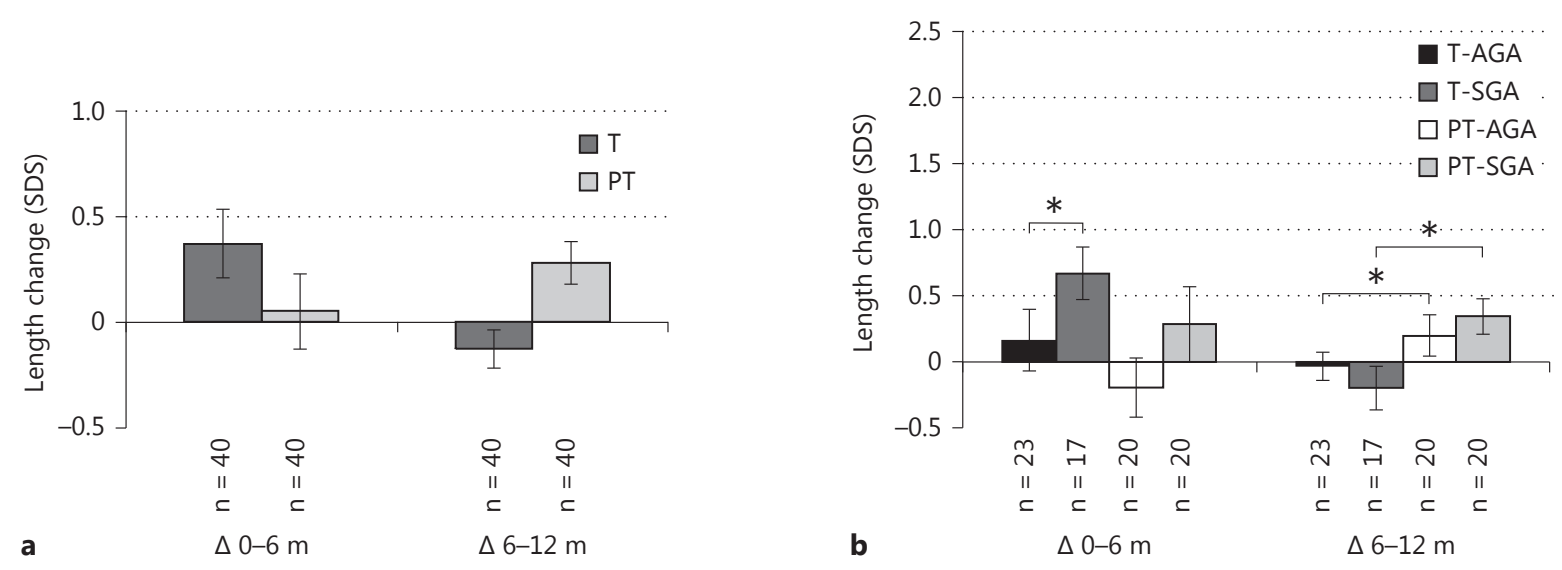

Fig. 2. Postnatal length SDS changes in the first semester $(\Delta 0-6 \mathrm{~m})$ and in the second semester $(\Delta 0-12 \mathrm{~m})$ of life, comparing the term (T) and preterm (PT) infants (a) and T and PT infants grouped according to by BW SDS (AGA or SGA) (b). Mean \pm SEM. ${ }^{*} \mathrm{p}<0.05$.

weight gain in term-SGA infants was significantly greater than that in term-AGA ones (fig. 1b). Between 6 and 12 months, weight gain SDS was greater in preterm than in term infants (fig. 1a): weight gain SDS was greater in preterm-SGA compared to that of term-SGA infants, but it was not different between preterm-AGA and term-AGA (fig. 1b).

Changes in Length over Time

There was no difference in linear growth SDS between term and preterm infants in either the 0-6- or 6-12-month interval (fig. 2a). We next evaluated the infants' linear growth according to both their gestational age and birth weight SDS (fig. 2b). In the 0-6-month period, the length change SDS in term-SGA was greater than that in term-AGA, but no difference between these two groups was found in the 6-12-month period. No difference in length change SDS was noted between preterm-SGA and preterm-AGA, either in the 0-6- or 6-12-month period. When we compared the length change SDS between preterm-AGA and term-AGA, and between preterm-SGA and term-SGA (i.e. different gestational age and similar birth weight SDS), we found no difference in the 0-6-month period. In contrast, in the 6-12-month period, the length change SDS in preterm-AGA and preterm-SGA was significantly greater than that of term-AGA and term-SGA, respectively (fig. 2b).

Fibroblast Growth Factor 21 and Growth in Infancy

\section{FGF21 Serum Levels}

Comparison of FGF21 Serum Levels between Infants Grouped According to Their Gestational Age and Birth Weight SDS

Mean \pm SD and IQR (q25-q75) of serum FGF21 concentrations from cord blood (cross-sectional group) and at 6 and 12 months (longitudinal group) are shown in figure 3. FGF21 was detectable in $93 \%$ of cord samples and in $100 \%$ of the 6 - and 12-month samples (standard curve range $16-1,000 \mathrm{pg} / \mathrm{ml}$ ).

At birth (cord blood), there was no difference in FGF21 levels between term and preterm (fig. 3a), or between AGA and SGA (term or preterm) (fig. $3 b$ ).

At 6 months, FGF21 in term infants was significantly greater than that of preterm infants (fig. 3a), while at 12 months there was no difference between groups. In cord blood the FGF21 level was significantly associated with leptin levels $(r=0.41, p<0.001)$, independent of gestational age or birth weight SDS. No association between FGF21 and leptin was detected at 6 or 12 months.

\section{Correlations between FGF21 Serum Levels and}

Changes in Weight and Length

First, we analyzed the correlation between changes of serum FGF21 and weight and length changes SDS in the 0-6-month period. In the whole cohort, we found a significant negative correlation between FGF21 and length change $(\mathrm{r}=-0.5, \mathrm{p}<0.05)$ and no correlation with the weight change. Next, we analyzed the correlation between 
Fig. 3. Serum FGF21 concentrations (median and IQR q25-q75) in the two groups of term (T) and preterm (PT) infants (a), and the T and PT infants grouped according to by BW SDS (AGA or SGA) (b). FGF21 was measured in the cord blood $(\mathrm{CB})$ at 6 months $(6 \mathrm{~m})$ and 12 months $(12 \mathrm{~m})$ of life. ${ }^{*} \mathrm{p}<0.05,{ }^{* *} \mathrm{p}<0.01$.

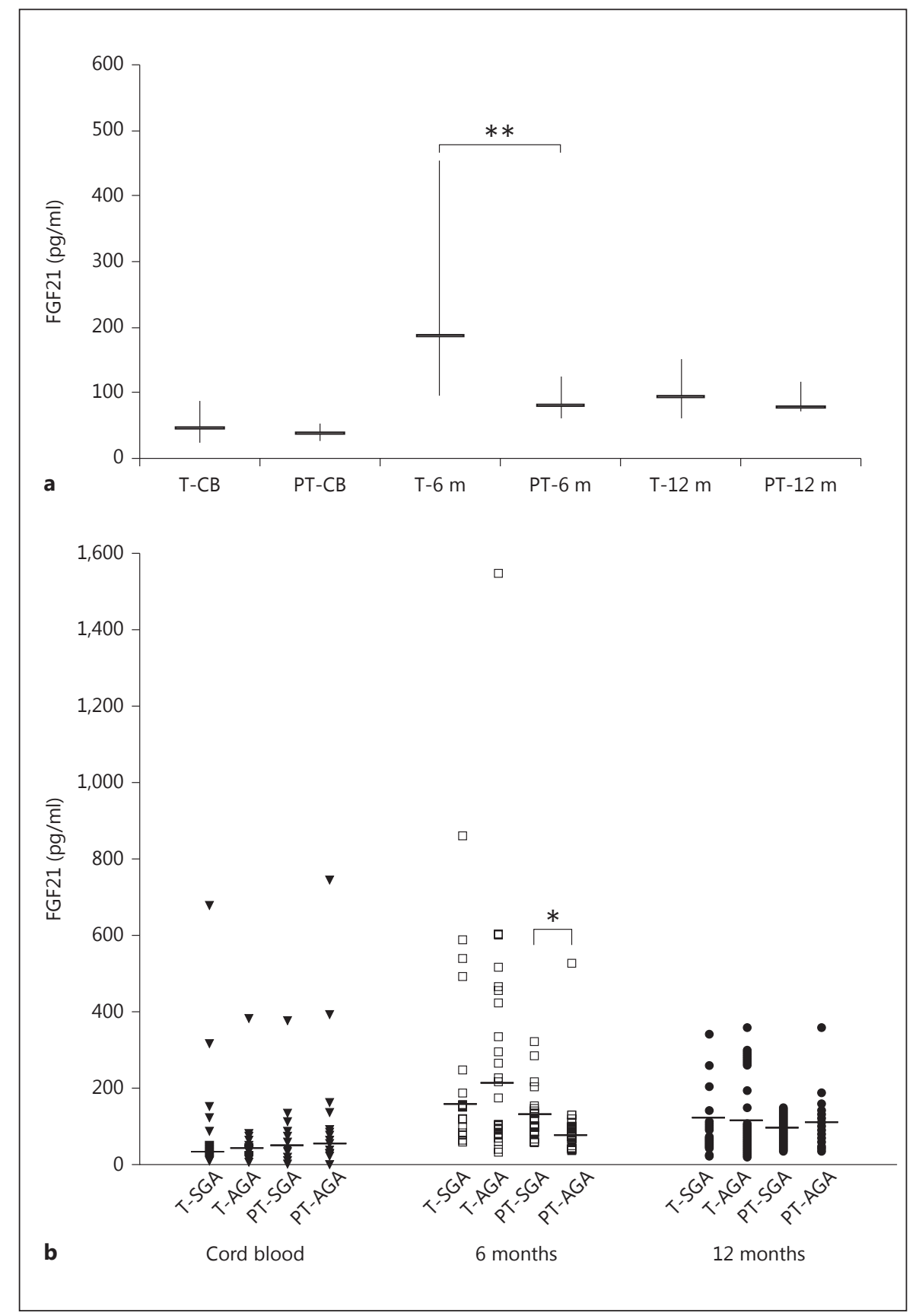

changes of FGF21 serum levels in the 6-12-month period and changes in anthropometric (weight SDS and length SDS) parameters in the same time period.

In the whole cohort, we found a significant positive correlation between serum FGF21 changes and weight SDS changes during the 6-12-month period $(\mathrm{r}=0.29, \mathrm{p}=0.01)$. While in this period there was no correlation between FGF21 and length SDS changes in the whole cohort $(r=$
$-0.19, \mathrm{p}=0.08$ ), we found a significant negative correlation between FGF21 changes and length SDS changes in the preterm-AGA subgroup $(\mathrm{r}=-0.45, \mathrm{p}<0.04)$ (fig. 4).

Furthermore, we analyzed the correlation between serum FGF21 level and catch-up in length. Length catch-up (increase in length $\geq 0.67 \mathrm{SD}$ ) in the $0-12$ month period was inversely associated to FGF21 at 12-months ( $\mathrm{r}=$ $-0.22, \mathrm{p}<0.05)$. The association almost reached statistical 
Fig. 4. Correlation (Spearman's) of FGF21 changes $(\mathrm{pg} / \mathrm{ml})$ and length SDS changes (SDS) during the 6-12-month period in the preterm-AGA subgroup $(\mathrm{n}=20) . \mathrm{r}=-0.45$, $\mathrm{p}<0.04$.

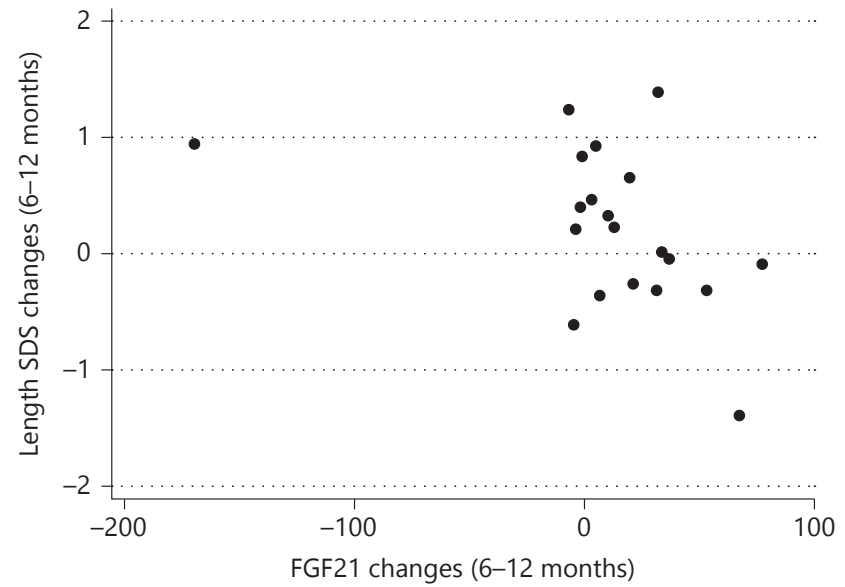

FGF21 changes (6-12 months) significance at 6 months $(\mathrm{r}=-0.22, \mathrm{p}=0.05)$. Those infants who performed length catch-up had a lower concentration of FGF21 (84.4 \pm 8.1 vs. $118.4 \pm 86 \mathrm{pg} / \mathrm{ml}, \mathrm{p}<$ 0.05) (fig. 5).

In the $0-6$-month period, $25 \%$ of all infants in the longitudinal group experienced length catch-up ( 9 termSGA, 6 term-AGA, 7 preterm-SGA, 3 preterm-AGA). In term infants with length catch-up, FGF21 at 6 months was significantly lower than in term infants without length catch-up (102.3 pg/ml, range 89.2-227.1 vs. 293.7 $\mathrm{pg} / \mathrm{ml}$, range $115.9-489.7, \mathrm{p}<0.05)$.

In the $6-12$-month period, 30 infants $(37.5 \%$ of the longitudinal group) underwent length catch-up, 14 were born term ( 7 term-SGA/7 term-AGA) and 16 were born preterm (12 preterm-SGA/4 preterm-AGA). In all infants with length catch-up in the 6-12-month period, median (IQR) serum FGF21 change (decrease) was greater than that seen in infants without length catch-up $(21.9 \mathrm{pg} / \mathrm{ml}$, range -0.7 to 62.4 vs. $6.5 \mathrm{pg} / \mathrm{ml}$, range -17.5 to 169 ), but the difference did not reach statistical significance $(\mathrm{p}=$ 0.07). However, in the subgroup of term infants with length catch-up, the FGF21 decrease was significantly greater than that of term infants without length catch-up (117.8 pg/ml, range $13.3-428$ vs. $27.6 \mathrm{pg} / \mathrm{ml}$, range $0.0 / 131.8, \mathrm{p}<0.05)$. No difference was found in the preterm group.

Lastly, we developed a mixed model with repeated measures to analyze the evolution of length SDS changes during the 0-12-month period, based on serum concentrations of insulin, leptin, and FGF21 (online suppl. table; www.karger.com/doi/10.1159/000367922). Each unit of insulin $(\mu \mathrm{IU} / \mathrm{ml})$ concentration at 12 months and the increase in insulin concentrations (6-12 months) was as-

Fibroblast Growth Factor 21 and Growth in Infancy

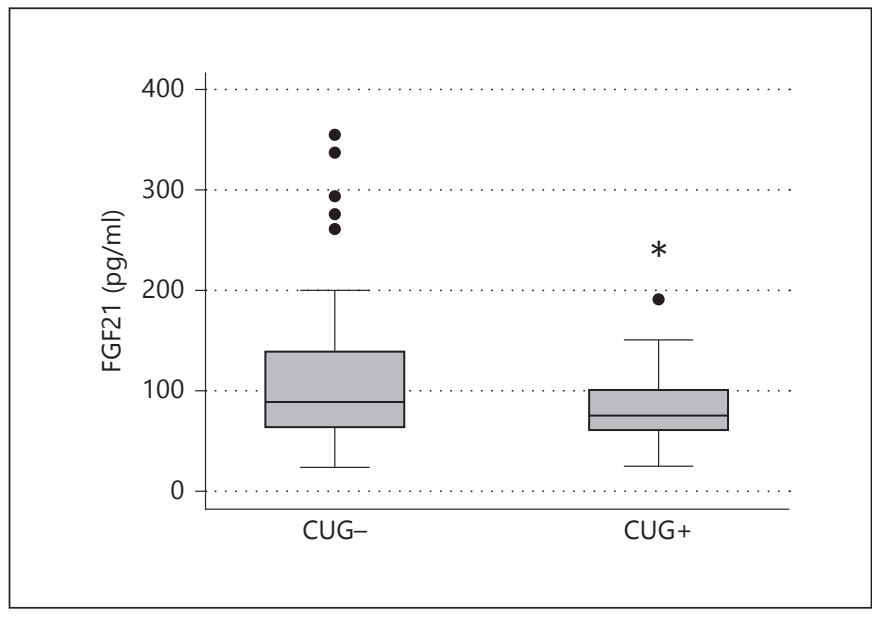

Fig. 5. Serum FGF21 concentrations (median and IQR q25-q75) in infants who performed length catch-up (CUG+) versus those who did not perform catch-up growth (CUG-). ${ }^{*} \mathrm{p}<0.05$.

sociated with a shorter length SDS of $0.536(\mathrm{p}<0.01)$ and $0.027(<0.05)$ units, respectively; each unit of increase in leptin concentrations at 12 months was associated with an increase in length SDS of $0.024(\mathrm{p}<0.001)$.

\section{Discussion}

To the best of our knowledge, this is the first study aimed at analyzing the correlation between longitudinal change of serum FGF21 levels during the first year of life, linear growth, and other metabolic factors in infants born at different gestational ages and birth weight SDS. 
FGF21 acts both as an endocrine factor and as a paracrine growth factor. Experimental evidence in rodents has previously suggested that FGF21 may contribute to regulate bone growth. Transgenic mice overexpressing FGF21 exhibit a smaller size and shorter tibial bones [3]. Furthermore, mice undergoing sustained food restriction experience reduced bone growth and increased FGF21 expression in the liver and in the growth plate [12]; in contrast, FGF21 knockout mice exposed to the same degree of food restriction are characterized by spared bone growth [13]. Based on these findings, it would appear that FGF21 is a negative regulator of bone growth.

In our longitudinal cohort, we found a substantially similar linear growth rate in the first 6 months of life when we compared term and preterm infants; in contrast, in the 6-12-month period, preterm infants grew significantly faster than term infants, and both preterm-SGA and preterm-AGA grew more than term-SGA and termAGA, respectively. In other words, linear growth of term infants progressively decelerated while that of preterm infants accelerated during the first year of life.

At birth (cord blood), there is no difference in FGF21 levels between term and preterm, or between AGA and SGA, suggesting that length of gestation and prenatal growth may not be associated with FGF21 secretory pattern and/or action. Another possible explanation is that perinatal factors, such as those related to the 'stress' of delivery, may neutralize any possible differences in FGF21 serum levels occurring prenatally.

In the 0-6-month period, in the whole cohort of infants, serum FGF21 was inversely correlated to the length change SDS (i.e. greater linear growth was associated with lower serum FGF21), and such a significant inverse correlation persisted in the preterm-AGA group in the 6-12-month period. Consistent with this finding, term infants experiencing catch-up growth in the first 6 months of life exhibited lower serum FGF21 levels at 6 months, and those with catch-up growth between 6 and 12 months had a greater decrease of serum FGF21 level in the same time period.

Regarding the mechanisms linking lower FGF21 levels and an accelerated linear growth, previous evidence implicates a possible interaction between insulin sensitivity and FGF21 expression. In adults, serum FGF21 concentrations are positively associated with serum insulin and HOMA-IR, thus suggesting a positive correlation with insulin-resistant states $[14,15]$. In our cohort, at 12 months of age, serum insulin levels were significantly associated with shorter length SDS, suggesting that hyperinsulinemia secondary to insulin resistance is correlated with reduced linear growth in infancy. However, we found no correla- tion between serum FGF21 and insulin levels, either at 6 or at 12 months of age. With respect to the effects of FGF2 1 on bone growth, our previous studies in animals implicate a FGF21-dependent GH-resistant state as a causative mechanism of reduced bone growth $[13,16]$.

In support of these animal findings, in adults with anorexia nervosa, FGF21 is directly correlated with serum GH and inversely correlated with IGF-I levels [5]. We did not assess IGF-I in our cohort and thus the relationship of FGF21 with IGF-I remains speculative.

This study has several strengths. First, these observations were carried out in a cohort of term and preterm healthy children from a well-defined area in Santiago, Chile. As a consequence of the strong demographical stratification in this city, our study is an excellent indicator of ethnical and socioeconomical homogeneity [17]. Second, we were able to prospectively monitor anthropometric and laboratory data in a cohort of infants followed for the first year of their life.

The study has limitations as well. We were not able to obtain cord or early postnatal blood samples in the infants followed longitudinally in order to determine whether differences in FGF2 1 levels detected at 6 months of age were already present at birth. In addition, our sample included a relatively small number of subjects.

In conclusion, our findings suggest that circulating FGF21 levels are inversely correlated with linear growth velocity in infants during their first year of life, and such correlation does not depend on gestational age or birth weight SDS. Due to the importance of FGF21 as a metabolic regulator during states of undernutrition, our findings suggest a causative role for FGF21 in nutritional stunting, which is the most common type of growth failure in the world. Yet, these findings do not enable us to shed light on the hormonal/molecular mechanisms linking FGF21 action and linear growth in humans. We believe that future studies based on a larger population sample followed since birth, and including the evaluation of a wider range of growth-regulating factors and more specific markers of insulin sensitivity, are warranted.

\section{Acknowledgment}

This study was supported by Fondecyt 1090028 and 1110240 .

\section{Disclosure Statement}

The authors have no conflicts of interest to disclose. 


\section{References}

1 Domouzoglou EM, Maratos-Flier E: Fibroblast growth factor 21 is a metabolic regulator that plays a role in the adaptation to ketosis. Am J Clin Nutr 2011;93:901S-905S.

$>2$ Kharitonenkov A, Larsen P: FGF21 reloaded: challenges of a rapidly growing field. Trends Endocrinol Metab 2011;22:81-86.

$>3$ Inagaki T, Lin VY, Goetz R, Mohammadi M, Mangelsdorf DJ, Kliewer SA: Inhibition of growth hormone signaling by the fasting-induced hormone FGF21. Cell Metab 2008;8: 77-83.

4 Mraz M, Bartlova M, Lacinova Z, Michalsky D, Kasalicky M, Haluzikova D, Matoulek M, Dostalova I, Humenanska V, Haluzik M: Serum concentrations and tissue expression of a novel endocrine regulator fibroblast growth factor-21 in patients with type 2 diabetes and obesity. Clin Endocrinol (Oxf) 2009;71:369375.

5 Fazeli PK, Misra M, Goldstein M, Miller KK, Klibanski A: Fibroblast growth factor-21 may mediate growth hormone resistance in anorexia nervosa. J Clin Endocrinol Metab 2010; 95:369-374.

6 Dostalova I, Kavalkova P, Haluzikova D, Lacinova Z, Mraz M, Papezova H, Haluzik M: Plasma concentrations of fibroblast growth factors 19 and 21 in patients with anorexia nervosa. J Clin Endocrinol Metab 2008;93: 3627-3632.
7 Pittaluga E, Vernal P, Llanos A, Vega S, Henrriquez MT, Morgues M, Escobar M, Diaz A, Standen J, Moncada P, Arriagada M, Rodriguez L, Mericq V: Benefits of supplemented preterm formulas on insulin sensitivity and body composition after discharge from the neonatal intensive care unit. J Pediatr 2011;159:926-932. e922.

8 Juez G: Intrauterine growth curve for the appropriate diagnosis of intrauterine growth retardation (in Spanish). Rev Med Chil 1989; 117:1311.

-9 Pitaluga E, Vernal P, Mena P, Figueroa A, Henriquez MT, Toha D: Curva de crecimiento intrauterino para prematuros entre 23 a 36 semanas de edad gestacional (in Spanish). Rev Chil Pediatr 2002;73:135-141.

10 De Onis M, Garza C, Victora CG, Onyango AW, Frongillo EA, Martines J: The WHO multicentre growth reference study: planning, study design, and methodology. Food Nutr Bull 2004;25:S15-S26.

11 Soto N, Bazaes RA, Pena V, Salazar T, Avila A, Iniguez G, Ong KK, Dunger DB, Mericq MV: Insulin sensitivity and secretion are related to catch-up growth in small-for-gestational-age infants at age 1 year: results from a prospective cohort. J Clin Endocrinol Metab 2003;88:3645-3650.
2 Kubicky RA, Wu S, Kharitonenkov A, De Luca F: Role of fibroblast growth factor 21 in undernutrition-related attenuation of growth in mice. Endocrinology 2012;153:2287-2295.

$13 \mathrm{Wu} \mathrm{S}$, Levenson A, Kharitonenkov A, De Luca F: Fibroblast growth factor 21 inhibits chondrocyte function and growth hormone action directly at the growth plate. J Biol Chem 2012;287:26060-26067.

14 Semba RD, Sun K, Egan JM, Crasto C, Carlson OD, Ferrucci L: Relationship of serum fibroblast growth factor 21 with abnormal glucose metabolism and insulin resistance: The Baltimore Longitudinal Study of Aging. J Clin Endocrinol Metab 2012;97:1375-1382.

15 Lee Y, Lim S, Hong ES, Kim JH, Moon MK, Chun EJ, Choi SI, Kim YB, Park YJ, Park KS, Jang HC, Choi SH: Serum FGF21 concentration is associated with hypertriglyceridaemia, hyperinsulinaemia and pericardial fat accumulation, independently of obesity, but not with current coronary artery status. Clin Endocrinol (Oxf) 2014;80:57-64.

16 Kubicky RA, Wu S, Kharitonenkov A, De Luca F: Role of fibroblast growth factor 21 in undernutrition-related attenuation of growth in mice. Endocrinology 2012;153:2287-2295.

-17 Cifuentes L, Valenzuela CY, Cruz-Coke R, Armanet L, Lyng C, Harb Z: Genetic characterization of the hospital population of Santiago, Chile (in Spanish). Rev Med Chil 1988; 116:28-33. 